\title{
Socioeconomic status and health in the second half of life: findings from the German Ageing Survey
}

\author{
Ina Schöllgen • Oliver Huxhold • \\ Clemens Tesch-Römer
}

Received: 4 November 2008/Accepted: 11 January 2010/Published online: 4 February 2010

(C) Springer-Verlag 2010

\begin{abstract}
This study examined social inequalities in health in the second half of life. Data for empirical analyses came from the second wave of the German Ageing Survey (DEAS), an ongoing population-based, representative study of community dwelling persons living in Germany, aged 40-85 years $(N=2,787)$. Three different indicators for socioeconomic status (SES; education, income, financial assets as an indicator for wealth) and health (physical, functional and subjective health) were employed. It could be shown that SES was related to health in the second half of life: Less advantaged persons between 40 and 85 years of age had worse health than more advantaged persons. Age gradients varied between status indicators and health dimensions, but in general social inequalities in health were rather stable or increasing over age. The latter was observed for wealth-related absolute inequalities in physical and functional health. Only income-related differences in subjective health decreased at higher ages. The amount of social inequality in health as well as its development over age did not vary by gender and place of residence (East or West Germany). These results suggest that, in Germany, the influence of SES on health remains important throughout the second half of life.
\end{abstract}

Keywords Health - Social inequality · Second half of life - Cumulative advantage . Age-as-leveller · Continuity

Responsible editor: D. J. H. Deeg.

I. Schöllgen $(\bowtie) \cdot$ O. Huxhold · C. Tesch-Römer German Centre of Gerontology, Berlin, Germany e-mail: ina.schoellgen@dza.de

\section{Introduction}

Consistently, it has been shown that lower socioeconomic status (SES) is related to worse health (e.g. Adler et al. 1994; Mackenbach et al. 1997; Marmot et al. 1997). From a life span developmental perspective it is important to explore whether the strength of this relationship varies with age (Alwin and Wray 2005). Originally, studies examining social inequalities in health rarely paid attention to older people, but now evidence is mounting that SES plays a role for health in later life (e.g. Avendano et al. 2005; Berkman and Gurland 1998; Huisman et al. 2005; Pérès et al. 2005). Using data from the representative German Ageing Survey (DEAS), the present article addresses the question how the SES-health relationship develops over the adult life span.

Dynamics of social inequalities in health across the life span

Three contradictory theoretical assumptions concerning the influence of SES over the life span have been discussed in the literature (O'Rand and Henretta 1999). Proponents of cumulation theory (e.g. Dannefer 1987; Ross and Wu 1996) assume that the influence of SES on health increases continuously with age due to a socially stratified cumulation of resources as well as risks over the life span leading to a cumulative advantage or disadvantage. In the context of minority ageing the double jeopardy hypothesis states that age-related losses in resources amplify the effects of race or SES on health (Dowd and Bengtson 1978; Ferraro and Farmer 1996).

In contrast, representatives of the age-as-leveller hypothesis suggest that the strength of the SES-health relationship decreases in old age relative to middle adulthood due to a variety of factors. First, retirement may end 
inequalities in the work context, and social policies may lead to less inequality in old age. Second, biological frailty could account for an accelerated health decline of high SES people in old age leading to a convergence of the status groups (Herd 2006). Third, selective survival might also eliminate socioeconomic differences in health in later life (Lynch 2003). Finally, it has been suggested that the influence of SES on health in the second half of life is characterised by continuity (O'Rand and Henretta 1999). This perspective assumes that one's status in earlier life still exerts an influence in later life and that SES continuously shapes life chances and activities in old age.

\section{Inconsistencies in empirical results}

Research has provided empirical evidence for all three potential age gradients mentioned above. A decrease of socioeconomic differences in morbidity and mortality in old age supporting the age-as-leveller hypothesis has been found by many investigators (e.g. Beckett 2000; Herd 2006; House et al. 1994; Marmot and Shipley 1996). There is, however, also evidence for continuity of social inequalities in health (Marmot and Fuhrer 2004; Rostad et al. 2009; Yao and Robert 2008), and support for an increasing impact of SES on health over the life span (Kim and Durden 2007; Ross and Wu 1996). The use of different SES and health indicators may be one reason for inconsistent results across studies.

Measuring the socioeconomic status of older adults is particularly difficult. Each of the most widely used indicators-education, income and occupation-entails problems (Grundy and Holt 2001). Robert and House (1996) suggested that indicators of wealth like financial assets are more appropriate indicators for older people's SES and reflect cumulative processes better. Moreover, it is increasingly recognised that different SES facets have different meanings and indicate access to different personal resources (e.g. education implies knowledge about health and health behaviour, income indicates the ability to purchase health services). Therefore, different indicators of SES cannot be used interchangeably (Geyer and Peter 2000). In addition, SES might have a different meaning for women and men. Gender might influence the association between SES and health and its development over age for various reasons, such as differential participation in the labour force (Broese van Groenou et al. 2003; Huisman et al. 2003; Lampert 2000).

Moreover, it has long been recognised that health is a multidimensional construct as well. According to Liang (1986) there are three related but distinct aspects: the physical aspect (absence of disease), the functional aspect (capacity for task performance) and the subjective evaluation of one's health (taking into account more of the psychological aspect). Studies that address different health dimensions suggest that social inequalities might develop differently according to the health indicator considered. As Lampert (2000) has shown, for example, using a sample aged 70 to $100+$ years, small socioeconomic differences in multimorbidity (physical aspect of health) up to the age of 90 were followed by significant differences in the group of $90+$ years. A contrasting picture emerged for functional health where socioeconomic differences were significant at age 70-79 and disappeared in the older age groups.

Furthermore, there might be differences between countries in the development of social inequalities in health over the life span due to different health insurance regimes and differences in the extent of social inequalities. As has been already mentioned, most studies seem to find decreasing influences of SES on health in old age, but the majority of these studies have been conducted in the United States and Great Britain. It is not clear whether these results also hold for Germany. One study using several SES and health indicators and a sample limited to an age range of 60 years and older showed only slight age variation in the effect of SES on health in Germany, supporting the continuity hypothesis (Knesebeck et al. 2003).

Germany, however, has a unique history. Between 1949 and 1990, there existed two German states with distinct differences in political and economic structure. Hence, place of residence, i.e. living in East Germany or West Germany, might be related to health in later life. On average, residents of the former East experienced lower standards of living and a worse health care system relative to those living in the former West. Differences in the treatment of diseases, related to the quality of the health care system, may partly explain differences in more distal health outcomes such as mortality and subjective health that have been reported (Lüschen et al. 1997). Although a general health advantage for those living in West Germany is under debate (e.g. Mielck et al. 2000), two studies found that older East Germans report worse health than their western counterparts, which has been attributed to an overall unfavourable situation for older people in the former East (Hillen et al. 2000; Lüschen et al. 1997). Of special interest in this context are effects of place of residence on socioeconomic differences in health. In communist societies such as the former East Germany, for example, income had been distributed more equally and was less important for the access to goods than in West Germany. Thus, some studies found income-related health inequalities to be larger in West Germany (Mielck et al. 2000). As the studies mentioned here have been conducted shortly after the German reunification, an interesting question is whether differences in health as well as in the amount of social inequality in health between East and West Germany are still observed in more recent studies. 
The present study

Thus far, there are comparatively few studies on the development of social inequalities in health in later life, especially in Germany. Moreover, empirical research has provided inconsistent results. We assume that some of the empirical ambiguity is due to between-study differences in the SES and health indicators used. Therefore, the present study examines the linkages of three SES indicators (education, income, financial assets as an indicator for wealth) to three health aspects (physical, functional and subjective health). By using data from a nationally representative study covering a broad age range (40-85 years) the analyses allow a comprehensive understanding of the specific German situation regarding social health inequalities in the second half of life. We investigate the association between SES and health in relation to age in order to determine whether social inequality in health is characterised by an increase, decrease or stability across adult development. An increase in the strength of the SES-health association is expected if the cumulative disadvantage or the age as double jeopardy hypothesis is true. A decreasing influence of SES on health is expected if the ageas-leveller hypothesis is true. Finally, the continuity hypothesis predicts only little age differences or stability in the SES-health relationship. Theoretical assumptions suggest that gender and place of residence might influence the association between SES and health and its development. Thus, our analyses account for these influences.

\section{Methods}

Sample

Data for empirical analyses came from the second wave of the German Ageing Survey, an ongoing population-based, representative study of community dwelling persons living in Germany, aged 40-85 years. Data collection took place in 2002 and occurred via in-home interviews and additional self-administered questionnaires. The sample was drawn by means of national probability sampling. Here, only those respondents were included who completed both interview and questionnaire $(N=2,787)$, which is $90.4 \%$ of the original sample. Selectivity analyses according to Lindenberger et al. (2002) indicated that selectivity effects for all variables in this study were very small $(d<0.20)$.

The sample was systematically stratified by gender, place of residence (about one-third from East Germany) and age group (about equal proportions of 40-54, 55-69 and 70-85-year-old participants; Engstler and Wurm 2006). The group of 40-54-year-old people represents those that are predominantly part of the labour force. The 55-69-year-old people are primarily situated shortly before, at or shortly after the transition to retirement, whereas the oldest age group (70-85 years old) represents those that have retired some time ago or the "young old" (e.g. Baltes and Smith 2003). Information about the sample, by age group and in total, can be found in Table 1 . Comparing the age groups, it can be seen that especially in the oldest age group a larger proportion of the sample had no partner, low education, low income (with a non-linear age trend regarding financial assets), and poor physical, functional and subjective health.

\section{Measures}

\section{Socioeconomic and demographic indicators}

Level of education, income and financial assets were used as SES indicators. Participants reported their highest level of completed school education with reference to the German education scheme. Due to the limited extent of differentiation in the oldest age group where $75 \%$ did not obtain any degree or had left school at the compulsory level, only two levels of education were distinguished: low (corresponding to less than 10 years of school education) and medium to high (at least 10 years of school education).

Respondents provided the total net income per month for the household. To adjust for household size, this was divided by the weighted number of household members according to the new OECD scale ${ }^{1}$ (Figini 1998). Income was divided in tertiles for the analyses.

Respondents specified the amount of financial assets owned by them or their partners, including bank accounts, life-insurances and stocks, but excluding real estate. Three categories were distinguished (low: up to 5,000 €, medium: $5,000 €$ - up to $25,000 €$, high: $25,000 €$ or more) that divided the sample in roughly equal proportions.

Age was used as a continuous variable but also split into the three groups (40-54 years, 55-69 years and 70-85 years) for some of the analyses. Gender $(1=$ men, $2=$ women $)$ and place of residence $(1=$ West Germany, $2=$ East Germany $)$ were included in all analyses. Moreover, we controlled for partner status $(1=$ no partner, $2=$ partner $)$ to avoid spurious associations of SES and health (Murphy et al. 1997).

\section{Health indicators}

Comprehensive health measures were applied to include different aspects of health (Liang 1986). Physical health was assessed by using a checklist of 11 health problems (e.g. cardiovascular diseases, diabetes; see Appendix for

\footnotetext{
1 This scale assigns a value of 1 to the household head, of 0.5 to each additional adult member and of 0.3 to each child.
} 
Table 1 Sample characteristics by age group: percentage or mean

\begin{tabular}{|c|c|c|c|c|}
\hline Characteristics & $\begin{array}{l}40-54 \text { years } \\
n=959\end{array}$ & $\begin{array}{l}55-69 \text { years } \\
n=941\end{array}$ & $\begin{array}{l}70-85 \text { years } \\
n=887\end{array}$ & $\begin{array}{l}\text { Total } \\
N=2,787\end{array}$ \\
\hline Age (years) & 46.9 & 62.2 & 75.9 & 61.3 \\
\hline Female & 51.6 & 49.2 & 48.5 & 49.8 \\
\hline East Germany & 66.0 & 67.2 & 67.3 & 66.8 \\
\hline Partner & 86.6 & 83.7 & 61.0 & 77.5 \\
\hline \multicolumn{5}{|l|}{ School education } \\
\hline Low & 31.9 & 63.5 & 75.5 & 56.5 \\
\hline Medium to high & 68.1 & 36.5 & 24.5 & 43.5 \\
\hline \multicolumn{5}{|l|}{ Income } \\
\hline Low & 29.0 & 33.5 & 40.6 & 34.2 \\
\hline Medium & 29.1 & 32.8 & 35.9 & 32.5 \\
\hline High & 41.9 & 33.7 & 23.6 & 33.3 \\
\hline \multicolumn{5}{|l|}{ Financial assets } \\
\hline Low & 40.5 & 35.4 & 46.7 & 40.7 \\
\hline Medium & 31.5 & 38.5 & 32.2 & 34.1 \\
\hline High & 28.0 & 26.1 & 21.1 & 25.2 \\
\hline \multicolumn{5}{|l|}{ Physical health } \\
\hline Three or more diseases & 19.4 & 39.3 & 59.2 & 38.3 \\
\hline \multicolumn{5}{|l|}{ Functional health } \\
\hline Lowest quartile & 7.3 & 22.8 & 49.2 & 25.9 \\
\hline \multicolumn{5}{|l|}{ Subjective health } \\
\hline Less than good & 29.3 & 43.9 & 62.5 & 44.8 \\
\hline
\end{tabular}

the complete list). A sum score based on the absolute number of self-reported illnesses was computed for each person. Using a sum score has various advantages compared to the use of single self-reported illnesses, concerning, for example, parsimony and accordance between medical reports and self-reports (Ferraro and Farmer 1996; Katz et al. 1996). Furthermore, global scores of selfreported illnesses turned out to be a good predictor of 1year mortality (Chaudhry et al. 2005). Suffering from three or more diseases, a criterion that has been employed by other studies (e.g. Hewitt et al. 2003), was used as an indicator of poor physical health in this study. ${ }^{2}$

Functional health was measured by the subscale physical functioning of the SF-36, (version 1.0, Bullinger and Kirchberger 1998; Ware and Sherbourne 1992). Impairments in 10 activities (e.g. climbing stairs, walking several blocks) are rated on a three-point scale, higher values indicating less impairment. For the present analyses, belonging to the lowest quartile of the distribution indicated poor functional health (Sekine et al. 2006; Stansfeld et al. 2003).

\footnotetext{
2 Because a large portion (about $60 \%$ ) of our sample had two or more diseases, we did not use the criterion of two or more diseases (e.g. Avendano et al. 2005). This ensured comparability with the cut-off scores for the other two health indicators used here.
}

Subjective health was assessed by a single item asking "How do you assess your current state of health?" ( $1=$ very good to $5=$ very bad). Consistent with other studies, we used a rating of "less than good", i.e. having a value of three to five, as indicator for poor subjective health (e.g. Huisman et al. 2003; Kunst et al. 2005).

\section{Statistical analysis}

All analyses were done with Mplus version 5. To examine the association between SES and health, we firstly estimated logistic regression models, containing the SES indicator, age, gender, place of residence and partner status as predictors. In the models examining the effect of income and financial assets, we also controlled for education. SES variables were treated as categorical, the most advantaged group being the reference category. Odds ratios (ORs) and 95\% confidence intervals (CIs) were estimated.

Age differences in the association between SES and health were explored in several ways. First, we added interaction terms between age and the SES indicator to the logistic regression models and tested whether this improved model fit significantly (Tabachnick and Fidell 2007). A significant interaction suggests that relative differences in odds between the status groups vary by age. We also estimated absolute effects and absolute differences on a risk scale. For this purpose, we created dummy variables 
for the combination between SES and three age groups (40-54 years, 55-69 years and 70-85 years), treating the 40-54 year old with high SES as reference group. The odds ratios obtained from these models, again controlling for the covariates, were calculated into risk ratios (Zhang and $\mathrm{Yu}$ 1998). Finally, the risk ratios were multiplied with the prevalence rate in the reference group to estimate absolute effects, i.e. rates of poor physical, functional and subjective health by level of SES and age group. For education, we tested if rate differences between the two groups were significant for each age group and whether they varied between age groups. For income and financial assets, we tested if there was a significant linear increase in rates with decreasing SES and whether this effect differed between the age groups.

The interplay between SES, gender and place of residence, and age was examined by adding two- and threeway interaction terms to the regression models.

Single missing values were supplemented by data imputation with the expectation maximisation method (Dempster et al. 1977). Results were compared to those obtained by including only participants who provided complete data; the results were virtually identical.

We included the stratification variables age, gender and place of residence in all analyses. Methodological studies have shown that unbiased coefficients are obtained if variables on which sampling is based are included in the models, nullifying the need for sample weights (Winship and Radbill 1994; see also, Lynch 2003). Repeating analyses with weighted data yielded largely equivalent results.

\section{Results}

The association between SES and health over age

Results are presented for each SES indicator (education, income and financial assets) separately. Tables 2, 4 and 6 display ORs and 95\% CIs obtained with logistic regressions including the SES indicator and the covariates. Tables 3, 5 and 7 show rates and rate differences across age and SES groups controlled for covariates. Statistically significant effects $(p<0.05)$ are indexed by an asterisk. Whether or not interactions between SES and age were significant can be found in the last rows of Tables 2, 4 and 6 and in the last columns of Tables 3, 5 and 7. In the text, we also report marginally significant results $(p<0.10)$ with exact $p$ values.

\section{Education}

As can be seen in Table $2 \mathrm{a}$, education was significantly related to physical health, functional health and subjective health after adjusting for covariates $(p s<0.05)$. The interaction between education and age was not significant in any case (Table $2 b$ ).

Table 3 shows that level of education accounted for significant rate differences in poor physical and functional health in all age groups ( $p s<0.05)$. For subjective health level of education accounted for rate differences in the youngest and middle aged group only ( $p s<0.05$ ), and just failed to reach significance for the oldest age group $(p=0.07)$. The rate differences due to education did not vary between age groups in any case (interaction physical health: $B=-0.01, \mathrm{SE}=0.03$, n.s.; functional health: $B=0.03$, SE $=0.02$, n.s.; subjective health: $B=-0.02$, $\mathrm{SE}=0.03$, n.s.).

\section{Income}

Table $4 \mathrm{a}$ shows that after controlling for confounders, income was significantly related to functional health and subjective health $(p s<0.05)$ but not to physical health $(p=0.09$ for low income). The interaction between income and age was not significant for physical health and functional health, but was significant for subjective health $(p<0.05$, Table 4b). The interaction effect was mainly due to the decreased influence of low income on subjective health at higher ages.

Table 2 Education and health over age: results of the logistic regression models

\begin{tabular}{lccc}
\hline & Physical health & Functional health & Subjective health \\
\hline (a) Main effects: odds ratios (and 95\% CIs) of poor physical, functional and subjective health, by education and covariates \\
Gender (female) & $0.95(0.81-1.08)$ & $1.74 *(1.43-2.13)$ & $0.90(0.77-1.06)$ \\
Place of residence (East Germany) & $1.11(0.93-1.31)$ & $1.16(0.94-1.41)$ & $1.33 *(1.13-1.58)$ \\
Partner status (partner) & $0.88(0.72-1.08)$ & $0.69 *(0.55-0.87)$ & $0.74 *(0.61-0.90)$ \\
Age & $1.06^{*}(1.05-1.07)$ & $1.09 *(1.08-1.10)$ & $1.04 *(1.03-1.05)$ \\
Education (low) & $1.43 *(1.19-1.71)$ & $1.57 *(1.27-1.94)$ & $1.50 *(1.27-1.78)$ \\
(b) Interaction effect: interaction between education and age & & 0.17 (n.s.) \\
$\Delta \chi^{2}(1)$ & 0.26 (n.s.) &
\end{tabular}

$* p<0.05$ 
Table 3 Rates (\%) of poor physical, functional and subjective health in the German Ageing Survey $(N=2,787)$, by level of education and age group. Rate difference by age group
Note: controlled for gender, place of residence and partner status

$* p<0.05$

\begin{tabular}{|c|c|c|c|c|}
\hline & $\begin{array}{l}\text { Medium-high } \\
\text { education }\end{array}$ & Low education & $\begin{array}{l}\text { Difference between levels } \\
\text { of education }\end{array}$ & $\begin{array}{l}\text { Interaction: levels of } \\
\text { education by age }\end{array}$ \\
\hline \multicolumn{5}{|l|}{ Physical health } \\
\hline $40-54$ years & 16.3 & 26.9 & $10.6^{*}$ & \multirow{3}{*}{ n.s. } \\
\hline 55-69 years & 35.0 & 42.2 & $7.2^{*}$ & \\
\hline $70-85$ years & 51.7 & 60.6 & $8.9^{*}$ & \\
\hline \multicolumn{5}{|c|}{ Functional health } \\
\hline 40-54 years & 5.7 & 11.3 & $5.6^{*}$ & \multirow{3}{*}{ n.s. } \\
\hline 55-69 years & 17.7 & 26.6 & $8.9^{*}$ & \\
\hline $70-85$ years & 40.0 & 50.8 & $10.8 *$ & \\
\hline \multicolumn{5}{|c|}{ Subjective health } \\
\hline $40-54$ years & 26.5 & 37.1 & $10.6^{*}$ & \multirow{3}{*}{ n.s. } \\
\hline 55-69 years & 35.5 & 49.6 & $14.1^{*}$ & \\
\hline $70-85$ years & 56.2 & 63.1 & 6.9 & \\
\hline
\end{tabular}

Table 4 Income and health over age: results of the logistic regression models

\begin{tabular}{lccc}
\hline & Physical health & Functional health & Subjective health \\
\hline (a) Main effects: odds ratios (and 95\% CIs) of poor physical, functional and subjective health, by income and covariates \\
Gender (female) & $0.95(0.80-1.12)$ & $1.71 *(1.40-2.01)$ & $0.89(0.76-1.04)$ \\
Place of residence (East Germany) & $1.06(0.89-1.27)$ & $1.02(0.83-1.26)$ & $1.20^{*}(1.01-1.43)$ \\
Partner status (partner) & $0.89(0.73-1.10)$ & $0.72 *(0.57-0.90)$ & $0.77 *(0.63-0.94)$ \\
Education (low) & $1.35^{*}(1.11-1.64)$ & $1.33^{*}(1.06-1.67)$ & $1.31^{*}(1.09-1.57)$ \\
Age & $1.06^{*}(1.05-1.07)$ & $1.09^{*}(1.08-1.10)$ & $1.04 *(1.03-1.05)$ \\
Income (medium) & $1.11(0.89-1.38)$ & $1.28(0.98-1.66)$ & $1.27 *(1.03-1.55)$ \\
Income (low) & $1.22(0.97-1.52)$ & $1.78^{*}(1.36-2.31)$ & $1.63 *(1.31-2.01)$ \\
(b) Interaction effect: interaction between income and age & & 1.19 (n.s.) \\
$\Delta \chi^{2}$ (2) & 0.58 (n.s.) & $7.93 *$ \\
\hline
\end{tabular}

$* p<0.05$

For physical health, statistical tests revealed no significant linear increase in rates of poor health with decreasing income in any age group (Table 5) and no significant variation in rate differences due to income between age groups (interaction: $B=0.00, \mathrm{SE}=0.01$, n.s.). There was a significant linear increase in rates of poor functional health with decreasing income in the youngest and oldest age group $(p s<0.05)$, but not in the middle age group $(p=0.06)$. The effects due to income did not differ significantly between the age groups (interaction: $B=0.02$, $\mathrm{SE}=0.01$, n.s.). For subjective health, there was a significant linear increase in rates of poor health with decreasing income for the youngest and middle age group $(p s<0.05)$, but not for the oldest age group. The significant interaction $(B=-0.06, \mathrm{SE}=0.03, p<0.05)$ points to smaller rate differences in subjective health due to income in the oldest age group.

\section{Financial assets}

In the overall sample, physical health was largely unrelated to financial assets after controlling for confounders ( $p=0.07$ for low assets), as can be seen in Table 6a. In contrast, functional and subjective health were significantly associated with financial assets $(p s<0.05)$. For physical health, adding the interaction coefficients between financial assets and age to the regression did not significantly improve model fit (Table 6b). However, a post hoc test revealed that the positive interaction between low financial assets and age just failed to reach significance $\left(\Delta \chi^{2}(1)=3.32, p=0.07\right)$. For functional health and subjective health, adding the interaction terms hardly changed model fit (Table 6b).

Analyses of rates and rate differences are depicted in Table 7. For physical health, there was a significant linear increase in rates of poor health with decreasing financial 
Table 5 Rates (\%) of poor physical, functional and subjective health in the German Ageing Survey $(N=2,787)$, by level of income and age group. Rate difference by age group
Note: controlled for gender, place of residence, partner status and education

$* p<0.05$

\begin{tabular}{ccclll}
\hline & $\begin{array}{l}\text { High } \\
\text { income }\end{array}$ & $\begin{array}{l}\text { Medium } \\
\text { income }\end{array}$ & $\begin{array}{l}\text { Low } \\
\text { income }\end{array}$ & $\begin{array}{l}\text { Linear difference between } \\
\text { levels of income }\end{array}$ & $\begin{array}{l}\text { Interaction: levels } \\
\text { of income by age }\end{array}$ \\
\hline $\begin{array}{c}\text { Physical health } \\
\text { 40-54 years }\end{array}$ & 16.2 & 21.4 & 19.0 & 1.6 & \\
55-69 years & 33.3 & 32.5 & 40.0 & 3.3 & \\
70-85 years & 52.9 & 52.7 & 53.7 & 0.5 & \\
$\begin{array}{c}\text { Functional health } \\
\text { 40-54 years }\end{array}$ & 4.2 & 6.0 & 11.3 & $3.5^{*}$ & \\
55-69 years & 17.2 & 19.2 & 23.5 & 3.3 & \\
70-85 years & 35.7 & 40.8 & 48.1 & $6.4^{*}$ & \\
Subjective health & & & & & \\
40-54 years & 21.9 & 27.2 & 36.8 & $7.4^{*}$ & \\
55-69 years & 33.0 & 39.4 & 45.7 & $6.4^{*}$ & \\
$70-85$ years & 54.9 & 54.6 & 56.8 & 1.1 & \\
\hline
\end{tabular}

Table 6 Financial assets and health over age: results of the logistic regression models

\begin{tabular}{lccc}
\hline & Physical health & Functional health & Subjective health \\
\hline (a) Main effects: odds ratios (and 95\% CIs) of poor physical, functional and subjective health, by financial assets and covariates \\
Gender (female) & $0.94(0.79-1.11)$ & $1.66^{*}(1.36-2.04)$ & $0.86(0.73-1.02)$ \\
Place of residence (East Germany) & $1.07(0.90-1.24)$ & $0.99(0.81-1.22)$ & $1.20^{*}(1.01-1.42)$ \\
Partner status (partner) & $0.91(0.74-1.13)$ & $0.79^{*}(0.62-0.99)$ & $0.82(0.67-1.00)$ \\
Education (low) & $1.37 *(1.14-1.65)$ & $1.29^{*}(1.03-1.61)$ & $1.32^{*}(1.11-1.58)$ \\
Age & $1.06^{*}(1.05-1.07)$ & $1.09^{*}(1.08-1.10)$ & $1.04^{*}(1.03-1.05)$ \\
Financial assets (medium) & $1.05(0.84-1.32)$ & $1.56^{*}(1.18-2.07)$ & $1.28^{*}(1.03-1.59)$ \\
Financial assets (low) & $1.23(0.98-1.55)$ & $2.62^{*}(1.99-3.46)$ & $1.91 *(1.54-2.38)$ \\
(b) Interaction effect: interaction between financial assets and age & & 1.51 (n.s.) \\
$\Delta \chi^{2}$ (2) & 3.87 (n.s.) & 0.50 (n.s.) \\
\hline
\end{tabular}

$* p<0.05$

Table 7 Rates (\%) of poor physical, functional, and subjective health in the German Ageing Survey $(N=2,787)$, by financial assets and age group. Rate difference by age group

Note: controlled for gender, place of residence, partner status, and education

$* p<0.05$

assets for the middle and oldest age group ( $p s<0.05)$, but not for the youngest age group. The significant interaction ( $B=0.03$, SE $=0.01, p<0.05$ ), pointed to larger absolute differences in poor physical health due to financial assets at higher ages. For functional health, there was a

\begin{tabular}{|c|c|c|c|c|c|}
\hline & $\begin{array}{l}\text { High } \\
\text { assets }\end{array}$ & $\begin{array}{l}\text { Medium } \\
\text { assets }\end{array}$ & $\begin{array}{l}\text { Low } \\
\text { assets }\end{array}$ & $\begin{array}{l}\text { Linear difference between } \\
\text { levels of assets }\end{array}$ & $\begin{array}{l}\text { Interaction: levels } \\
\text { of assets by age }\end{array}$ \\
\hline \multicolumn{6}{|l|}{ Physical health } \\
\hline 40-54 years & 19.8 & 19.4 & 17.9 & -0.9 & \multirow{3}{*}{$*$} \\
\hline 55-69 years & 33.5 & 34.8 & 38.6 & $2.4^{*}$ & \\
\hline $70-85$ years & 48.3 & 50.5 & 58.8 & $5.8^{*}$ & \\
\hline \multicolumn{6}{|c|}{ Functional health } \\
\hline 40-54 years & 3.4 & 5.6 & 10.3 & $3.9^{*}$ & \multirow{3}{*}{$*$} \\
\hline 55-69 years & 13.4 & 19.9 & 25.9 & $7.7 *$ & \\
\hline $70-85$ years & 30.9 & 38.0 & 53.4 & $11.4 *$ & \\
\hline \multicolumn{6}{|c|}{ Subjective health } \\
\hline 40-54 years & 22.8 & 26.1 & 34.0 & $5.8^{*}$ & \multirow{3}{*}{ n.s. } \\
\hline 55-69 years & 34.0 & 37.6 & 47.5 & $6.9^{*}$ & \\
\hline $70-85$ years & 45.0 & 54.7 & 63.9 & $9.4 *$ & \\
\hline
\end{tabular}

significant linear increase in rates of poor health with decreasing financial assets for all age groups $(p s<0.05)$. Again, the significant interaction indicates larger rate differences at higher ages $(B=0.04, \mathrm{SE}=0.01, p<0.05)$. Linear increases in rates of poor subjective health with 
decreasing financial assets were significant in each case $(p s<0.05)$ but did not vary between age groups (interaction: $B=0.03, \mathrm{SE}=0.03$, n.s.).

Effects of gender and place of residence

As can be seen in Tables 2, 4 and 6, gender was significantly related to functional health above and beyond the other predictors $(p s<.05)$. Further analyses (results not shown) revealed a tendency for stronger gender differences with increasing age to the disadvantage of older women (model with education: $p=0.08$, model with income: $p=0.09$, model with assets: $p=0.05$ ).

Moreover, people living in East Germany were more likely to report poor subjective health $(p s<0.05)$. For physical health as the outcome, there was a consistent significant interaction between age and place of residence ( $p$ s < 0.05). Age had a stronger influence for people living in East Germany than for their western counterparts. We did not find, however, stable and statistically significant interactions between SES and gender, SES and place of residence, or between SES, gender/place of residence and age on any health outcome (results not shown).

\section{Discussion}

This study examined social inequalities in health in the second half of life, using data from the German Ageing Survey. Socioeconomic status was related to health in the second half of life: In general, less advantaged persons between 40 and 85 years of age had worse health than more advantaged persons. Our analyses showed that age gradients of social inequalities in health vary between SES indicators (education, income, financial assets), health dimensions (physical, functional, subjective) and measure (absolute vs. relative differences). A summary of results is displayed in Table 8.
SES and health in the second half of life

Education had a stable effect on all three health measures across the adult life span both in terms of relative differences in odds and absolute or rate differences between the groups. Hence, for education, our results lend some support to the continuity hypothesis. The educational background continuously shapes life chances and activities and thus influences health across the entire life span, including old age.

Income was unrelated to physical health, exerted a stable influence across the second half of life on functional health, and had a decreasing influence on the subjective health of the oldest adults. Hence, for income, the results provide some evidence for the continuity hypothesis for functional health and the age-as-leveller hypothesis for subjective health. While it is not clear why this was only found for subjective health in this study, one reason for a decreasing influence of income with increasing age is probably that income does not adequately reflect the financial situation in old age (see also, Huisman et al. 2003).

To overcome this, we used wealth indicated by financial assets as an additional predictor. Wealth reflects cumulative processes and is thus a better indicator for the financial situation in later life (e.g. Robert and House 1996). For financial assets, we found a more complex picture. The association between financial assets and physical health increased with age, although the interaction only reached significance for absolute health inequalities (rate differences). The influence of financial assets on functional health was stable over age if one looks at relative differences in odds and increased with age if one looks at absolute or rate differences between the status groups. There was little age variation in the robust relationship between financial assets and subjective health. Hence, for financial assets, both cumulative (dis)advantage and continuity hypothesis seem plausible.

When analysing the age gradient in the association between financial assets and functional health, we obtained differential results depending on the measure, i.e. relative differences in odds versus absolute differences in rates. This
Table 8 Summary of results for the relation between socioeconomic status and health in the second half of life

\begin{tabular}{llll}
\hline & Physical health & Functional health & Subjective health \\
\hline $\begin{array}{l}\text { Education } \\
\text { Relative difference }\end{array}$ & Stability & Stability & \\
Absolute difference & Stability & Stability & Stability \\
Income & & & Stability \\
Relative difference & - & Stability & \\
Absolute difference & - & Stability & Decrease \\
Financial assets & & & Decrease \\
Relative difference & - & Stability & Stability \\
Absolute difference & Increase & Increase & Stability \\
\hline
\end{tabular}


difference is caused by the interaction of strong (relative) rate differences between asset groups at younger ages and the strong age effect on functional health. To illustrate this, we predicted age trends for poor functional and subjective health by combining the linear increases associated with age with the rate differences between financial asset groups at younger ages (mean age $=46$ years).

Figure 1 shows that these two factors lead to increasing absolute or rate differences in functional health (Fig. 1a) but not in subjective health (Fig. 1b), because in contrast to functional health the relative rate differences in subjective health at younger ages were smaller and the age trend less pronounced. In summary, although the relative increase of risk of poor functional health with age was the same for people belonging to different financial asset groups (continuity), looking at the larger absolute differences in rates at older ages one might still speak of a cumulation effect at the population level.
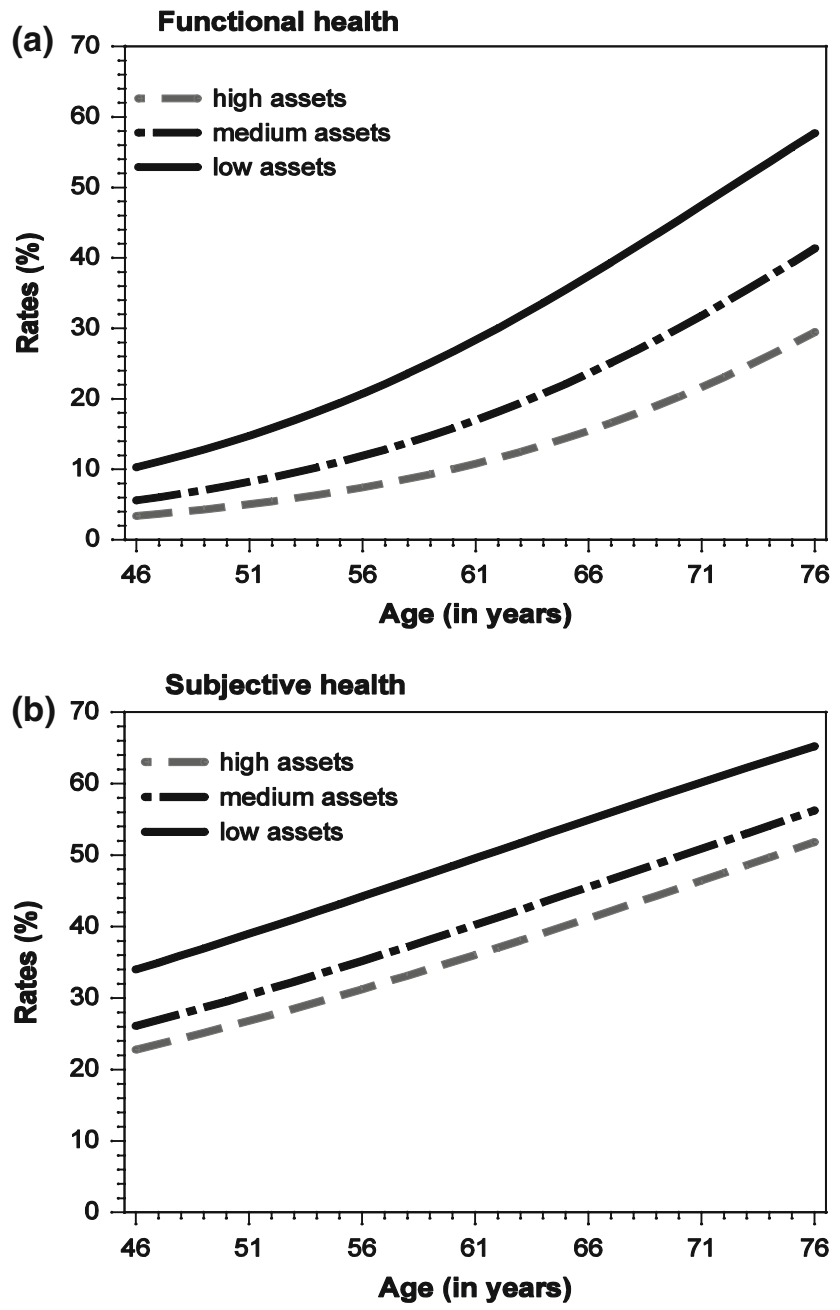

Fig. 1 Age trends in rates of poor functional health (a) and poor subjective health (b) for three groups with different amount of financial assets (low, medium, high)
These results fit into the broader discussion how to define and measure cumulative effects (Wilson et al. 2007). Ideally, one would have to take into account the duration of exposure to certain factors, which makes it more likely to find diverging health inequalities for income as well (Kim and Durden 2007). Moreover, as our results suggest, different perspectives, reflected by different measures, should be taken into account.

Continuity and cumulation, but no levelling off?

In contrast to other studies, our results point to the continuity and the cumulative (dis)advantage hypothesis (only the association between income and subjective health decreased with age). Why is this the case? First of all, we did not include a measure of (former) occupational status in the analyses. The age trend may differ for this indicator as occupationally based measures most closely represent working conditions, which might lose their relevance for health upon retirement. Secondly, the oldest participants were 85 years of age, thus our sample does not include the oldest old. As some of the processes being discussed as potential sources of decreasing inequalities in old age, i.e. biological frailty, may have a stronger impact in the most advanced ages, this might have prevented us from detecting such changes.

The third reason might be a theoretically substantial one. The finding of continuing social inequalities across the life span could be a consequence of the societal context. In contrast to the American health system, no change in the health insurance regime is associated with becoming 65 years in Germany. Furthermore, as there are larger social inequalities in health during midlife in the United States compared to Germany, the SES-health association in the USA might be attenuated in old age to a larger extent due to a stronger influence of selective mortality in earlier stages of life. These facts render the age-as-leveller hypothesis less likely in Germany (see also, Knesebeck et al. 2003). One has to keep in mind, however, that there are German studies as well as European-wide studies including German samples that find decreasing inequalities in health with increasing age for some health indicators and in some subsamples (e.g. Huisman et al. 2003; Lampert 2000).

\section{Effects of gender and place of residence}

Apart from replicating the well-known gender difference in functional health (e.g. Arber and Ginn 1993), which is especially pronounced at higher ages, we did not find any systematic effects of gender. Of special interest had been whether gender influences the amount of social inequality in health as well as its development over age, which was not the case. This is in line with other studies on this topic (e.g. Arber and Ginn 1993). In contrast, Huisman et al. 
(2003) found that the influence of SES decreased over age for women but not men. However, their sample was slightly older than ours, which might partly explain the inconsistencies between the studies.

We also looked at the effects of place of residence. Interestingly, more than a decade after the German reunification, we still found people living in East Germany to be more likely to report poor subjective health than those living in West Germany. We also found older people in East Germany to be particularly disadvantaged with regard to physical health. This replicates and extends results from studies that were conducted shortly after the political transition in 1989/90 (e.g. Hillen et al. 2000; Lüschen et al. 1997). Contrary to some of these studies, however, we did not find meaningful differences in the amount of health inequality and no differences in age trends according to place of residence. In former communist societies, such as East Germany, income had been distributed more equally and was not such an important indicator for access to goods. After the political transition, however, income inequalities became larger in East Germany, which might have reduced differences in the amount of income-related health inequality between East and West Germany in the present study.

\section{Limitations}

The cross-sectional nature of the data set limits the interpretation of the results. No stringent conclusions about causality can be made. Especially for income and financial assets as indicators of SES, it would have been equally plausible that deterioration in health exerts a negative influence on one's SES, at least for the younger groups. Although other studies suggest that these selection effects are not primarily responsible for social inequalities in health (e.g. Blane et al. 1993; Chandola et al. 2003), this effect cannot be ruled out completely. Moreover, cross-sectional analyses confound ageand cohort-effects, which might cancel each other out (Lynch 2003). In general, longitudinal data are preferable for investigating processes unfolding over the life span, which are underlying the hypotheses examined here.

Another limitation concerns the composition of the sample. The fact that institutionalised people are not included in the baseline samples of the DEAS limits the generalisability of the results. Moreover, as both poor health and low SES are linked to higher risk of institutionalisation (Gaugler et al. 2007), we probably underestimated SES differences in health in the oldest group (see also, Huisman et al. 2003).

A final concern regards the indicators used. Firstly, one might criticise the categorisation we used for the SES indicators. Concerning education, we distinguished only two levels as a large majority of the oldest age group had a low education. Regarding income, a study by Grundy and
Sloggett (2003) found more consistent relations to a variety of health indicators than we did. Their income measure distinguished recipients of income-support from non-recipients only. While this might be more meaningful than using tertiles, it is an indicator of poverty and thus ignores some of the health differences between status groups. Secondly, health was measured only by self-reports. It thus cannot be concluded without doubt that the present findings generalise to objective measures of physical health. A high accordance between self-reported health and physician-evaluated health has been shown, however, for physical health (e.g. Bush et al. 1989). Moreover, equally large educational inequalities in self-reported and performance-based measures of functional health and disability have been reported for older adults from the Netherlands (Huisman et al. 2005).

Addressing mechanisms: outlook on future analyses

Future research needs to examine the mechanisms underlying the association between SES and health in the second half of life. In this context, it should be considered that the influence of SES indicators varies by health aspect. Our results show that education was more consistently related to poor physical health than the financial indicators. Income, which was completely unrelated to physical health, was significantly related to functional and subjective health. We argue that this pattern of results is due to different mechanisms linking SES factors to varying aspects of health. Education exerts its influence on health via knowledge, attitudes towards health and health behaviour (e.g. Geyer and Peter 2000): These factors are relevant for the prevention of disease. In contrast, financial resources may be more important for dealing with and adapting to existing health problems. In line with this view, a study by House et al. (2005) showed that education was more important for the onset of health problems, whereas income and financial assets were more relevant for the progression of functional limitations. In addition, it should be analysed whether mechanisms relating SES to health differ over age. One of the few studies on this topic indicated that while health behaviour mediated the association between education and functional health in a group of 55-70-year-old people, for older people psychosocial factors became more relevant (Koster et al. 2006).

Acknowledgements This study was funded by the German Federal Ministry for Family, Senior Citizens, Women and Youth (Grant 3011720-2/2). The dissertation work of Ina Schöllgen is supported by the International Max Planck Research School "The Life Course: Evolutionary and Ontogenetic Dynamics" (LIFE).

\section{Appendix}

See Table 9. 
Table 9 The complete list of health problems assessed in the German Ageing Survey

\begin{tabular}{l}
\hline Cardiovascular diseases \\
Circulatory problems \\
Back or joint diseases \\
Diabetes \\
Gastro-intestinal diseases \\
Respiratory diseases \\
Cancer \\
Liver or kidney diseases \\
Bladder trouble \\
Diseases of the eye \\
Diseases of the ear
\end{tabular}

Dowd JJ, Bengtson VL (1978) Aging in minority populations: an examination of the double jeopardy hypothesis. J Gerontol 33:427-436

Engstler H, Wurm S (2006) Datengrundlagen und Methodik [Data base and method]. In: Tesch-Römer C, Engstler H, Wurm S (eds) Sozialer Wandel und individuelle Entwicklung in der zweiten Lebenshälfte. VS Verlag, Wiesbaden, Germany, pp 47-83

Ferraro KF, Farmer MM (1996) Double jeopardy to health hypothesis for African Americans: analysis and critique. J Health Soc Behav $37: 27-43$

Figini P (1998) Inequality measures, equivalence scales and adjustment for household size and composition. Luxembourg Income Study, Luxembourg

Gaugler JE, Duval S, Anderson KA, Kane RL (2007) Predicting nursing home admission in the U.S: a meta-analysis. BMC Geriatr 7:13-26

Geyer S, Peter R (2000) Income, occupational position, qualification and health inequalities - competing risks? (comparing indicators of social status). J Epidemiol Community Health 54:299-305

Grundy E, Holt G (2001) The socioeconomic status of older adults: how should we measure it in studies of health inequalities? J Epidemiol Community Health 55:895-904

Grundy E, Sloggett A (2003) Health inequalities in the older population: the role of personal capital, social resources and socio-economic circumstances. Soc Sci Med 56:935-947

Herd P (2006) Do functional health inequalities decrease in old age? Educational status and functional decline among the 1931-1941 birth cohort. Res Aging 28:375-392

Hewitt M, Rowland J, Yancik R (2003) Cancer survivors in the United States: age, health, and disability. J Gerontol Med Sci 58A:82-91

Hillen T, Schaub R, Hiestermann A, Kirschner W, Robra B-P (2000) Self rating of health is associated with stressful life events, social support and residency in East and West Berlin shortly after the fall of the wall. J Epidemiol Community Health 54:575-580

House JS, Lepkowski JM, Kinney AM, Mero RP (1994) The social stratification of aging and health. J Health Soc Behav 35:213234

House JS, Lantz PM, Herd P (2005) Continuity and change in the social stratification of aging and health over the life course: evidence from a nationally representative longitudinal study from 1986 to 2001/2002 (Americans' changing lives study). J Gerontol Soc Sci 60B:15-26

Huisman M, Kunst AE, Mackenbach JP (2003) Socioeconomic inequalities in morbidity among the elderly; a European overview. Soc Sci Med 57:861-873

Huisman M, Kunst AE, Deeg DJH, Grigoletto F, Nusselder W, Mackenbach JP (2005) Educational inequalities in the prevalence and incidence of disability in Italy and the Netherlands were observed. J Clin Epidemiol 58:1058-1065

Katz JN, Chang LC, Sangha O, Fossel AH, Bates DW (1996) Can comorbidity be measured by questionnaire rather than medical record review? Med Care 34:73-84

Kim J, Durden E (2007) Socioeconomic status and age trajectories of health. Soc Sci Med 65:2489-2502

Knesebeck Ovd, Lüschen G, Cockerham WC, Siegrist J (2003) Socioeconomic status and health among the aged in the United States and Germany: a comparative cross-sectional study. Soc Sci Med 57:1643-1652

Koster A, Bosma H, Broese van Groenou MI, Kempen GI, Penninx BW, van Eijk JT, Deeg DJH (2006) Explanations of socioeconomic differences in changes in physical function in older adults: results from the Longitudinal Aging Study Amsterdam. BMC Public Health 6:244

Kunst AE, Bos V, Lahelma E, Bartley M, Lissau I, Regidor E, Mielck A, Cardano M, Dalstra JAA, Geurts JJM, Helmert U, 
Lennartsson C, Ramm J, Spadea T, Stronegger WJ, Mackenbach JP (2005) Trends in socioeconomic inequalities in self-assessed health in 10 European countries. Int J Epidemiol 34:295-305

Lampert T (2000) Sozioökonomische Ungleichheit und Gesundheit im höheren Lebensalter: Alters- und geschlechtsspezifische Differenzen [Socioeconomic inequality and health in old age: Age and gender-specific differences]. In: Backes GM, Clemens W (eds) Lebenslagen im Alter: Gesellschaftliche Bedingungen und Grenzen. Leske + Budrich. Opladen, Germany, pp 159-185

Liang J (1986) Self-reported physical health among aged adults. J Gerontol 41:248-260

Lindenberger U, Singer T, Baltes PB (2002) Longitudinal selectivity in aging populations: separating mortality-associated versus experimental components in the Berlin Aging Study (BASE). J Gerontol Psychol Sci 57B:P474-P482

Lüschen G, Niemann S, Apelt P (1997) The integration of two health systems: social stratification, work and health in East and West Germany. Soc Sci Med 44:883-899

Lynch SM (2003) Cohort and life-course patterns in the relationship between education and health: a hierarchical approach. Demography 40:309-331

Mackenbach JP, Kunst AE, Cavelaars AEJM, Groenhof F, Geurts JJM (1997) Socio-economic inequalities in morbidity and mortality in Western Europe. Lancet 349:1655-1659

Marmot MG, Fuhrer R (2004) Socioeconomic position and health across midlife. In: Brim OG, Ryff CD, Kessler RC (eds) How healthy are we? A national study of well-being at midlife. The University of Chicago Press, Chicago, IL, pp 64-89

Marmot MG, Shipley MJ (1996) Do socioeconomic differences in mortality persist after retirement? 25 year follow up of civil servants from the first Whitehall study. BMJ 313:1177-1180

Marmot MG, Ryff CD, Bumpass LL, Shipley M, Marks NF (1997) Social inequalities in health: next questions and converging evidence. Soc Sci Med 44:901-910

Mielck A, Cavelaars AEJM, Helmert U, Martin K, Winkelhake O, Kunst AE (2000) Comparison of health inequalities between East and West Germany. Eur J Public Health 10:262-267

Murphy M, Glaser K, Grundy E (1997) Marital status and long-term illness in Britain. J Marriage Fam 59:156-164
O'Rand AM, Henretta JC (1999) Age and inequality: diverse pathways through later life. Westview Press, Boulder, CO

Pérès K, Jagger C, Lièvre A, Barberger-Gateau P (2005) Disabilityfree life expectancy of older French people: gender and education differentials from the PAQUID cohort. Eur J Ageing 2:225-233

Robert S, House JS (1996) SES differentials in health by age and alternative indicators of SES. J Aging Health 8:359-388

Ross CE, Wu C-L (1996) Education, age and the cumulative advantage in health. J Health Soc Behav 37:104-120

Rostad B, Deeg DJH, Schei B (2009) Socioeconomic inequalities in health in older women. Eur J Ageing 6:39-47

Sekine M, Chandola T, Martikainen P, Marmot MG, Kagamimori S (2006) Socioeconomic inequalities in physical and mental functioning of Japanese civil servants: explanations from work and family characteristics. Soc Sci Med 63:430-445

Stansfeld SA, Head J, Fuhrer R, Wardle J, Cattell V (2003) Social inequalities in depressive symptoms and physical functioning in the Whitehall II study: exploring a common cause explanation. J Epidemiol Community Health 57:361-367

Tabachnick BG, Fidell LS (2007) Using multivariate statistics. Pearson, Boston, MA

Ware JE, Sherbourne CD (1992) The MOS 36-item short-form health survey (SF-36): I. Conceptual framework and item selection. Med Care 30:473-483

Wilson AE, Shuey KM, Elder GH Jr (2007) Cumulative advantage processes as mechanisms of inequality in life course health. Am J Sociol 112:1886-1924

Winship C, Radbill L (1994) Sampling weights and regression analysis. Sociol Meth Res 23:230-257

Yao L, Robert SA (2008) The contribution of race, individual socioeconomic status, and neighborhood socioeconomic context on the self-rated health trajectories and mortality of older adults. Res Aging 30:251-273

Zhang J, Yu KF (1998) What's the relative risk? A method of correcting the odds ratio in cohort studies of common outcomes. JAMA 280:1690-1691 